\title{
Multiplexing Data and Control Channels in Random Access Underwater Networks
}

\author{
Stefano Basagni \\ ECE Department \\ Northeastern University \\ basagni@ece.neu.edu
}

\author{
Chiara Petrioli and Roberto Petroccia* \\ Dipartimento di Informatica \\ Università di Roma "La Sapienza" \\ \{petrioli,petroccia\}@di.uniroma1.it
}

\author{
Milica Stojanovic \\ ECE Department \\ Northeastern University \\ millitsa@ece.neu.edu
}

\begin{abstract}
We address random access networks with MAC protocols that use control packets such as RTS/CTS. These protocols reduce or eliminate collisions between data packets, but they typically remain prone to collisions between control and data packets. To avoid this type of collision, the data and control channels can be separated by multiplexing in the frequency domain. A small reduction in bandwidth is thus sacrificed in exchange for a reduced number of re-transmissions. This technique is investigated in conjunction with the distance-aware collision avoidance protocol (DACAP). Simulation results show that multiplexing offers some benefits to both throughput efficiency and energy consumption.
\end{abstract}

\section{INTRODUCTION}

Underwater wireless networking has been recognized as an enabling technology for a wide spectrum of applications that include ocean observation for scientific exploration or commercial exploitation, coastline protection, and prediction of underwater seismic and volcanic events [1]-[5]. The major challenges found in the design of underwater acoustic networks are the long propagation delay and low bandwidths. To address these issues, which are not normally present in terrestrial radio networks, research has been active on various topics in both deterministic and random access networks [6]. The focus of our present work is on the latter type of network, where a number of nodes (users) access the channel in the same bandwidth, submitting their requests randomly as the demand dictates. This type of channel sharing is suitable for situations where each node's traffic is bursty, consisting of packets that arrive at a sufficiently low rate that they do not require deterministic channel allocation (in fact, it would be wasteful to allocate the channel to a node that is not using it). The nodes access the channel using a medium access control (MAC) protocol whose task is to reduce the number of packet collisions, and strike a balance between information throughput and energy consumption.

A number of MAC protocols have recently been proposed to specifically address the long-delay low-

\footnotetext{
* Roberto Petroccia is also with the Department of Information Engineering of the University of Padova, Italy.
}

bandwidth problem of the acoustic channel [7]-[14]. Here, we focus on the Distance-Aware Collision Avoidance Protocol (DACAP) [10], which combines carrier sensing multiple access (CSMA) principles with medium access collision avoidance (MACA). This protocol uses dedicated control packets (request-to-send/clear-to-send (RTS/CTS) and short warning packets) to prevent collisions between data packets. However, while the absence of data/data collisions is guaranteed, control/data collisions are still possible. The effect of these collisions is often neglected, but, as we shall see, they can significantly harm the system performance.

In a wireless setting, it is possible (and common) for two nodes that cannot directly hear each other to impair each other's reception. Figure 1 illustrates such a situation. This situation is exacerbated in acoustic

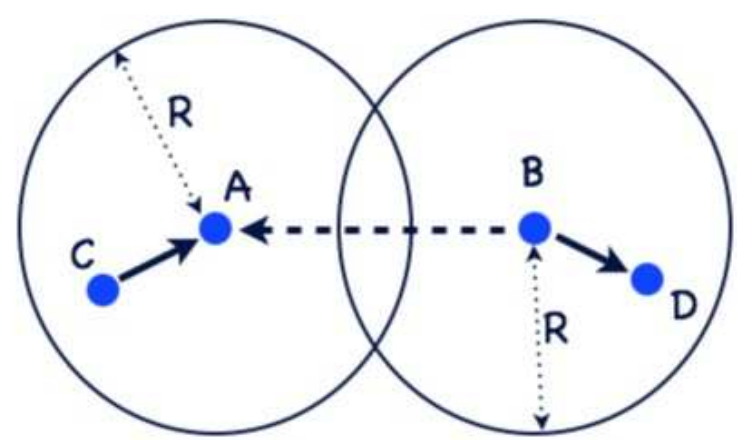

Figure 1. Although A and B are outside of each other's nominal range, B's transmission to D will reach A, interfering with reception from $\mathrm{C}$. The interference radius is thus greater than the nominal transmission radius $R$.

channels, where the spreading factor (path loss exponent) is low. In fact, through extensive simulations on most of the underwater MAC protocols proposed so far, we have observed that the vast majority of packet losses are due to this type of interference [14]. Specifically, in the case of RTS/CTS-based access à la IEEE 802.11 with the distributed coordination function, we observed that $90 \%$ of packet losses are due to interference coming from nodes that are out of the receiver's transmission 
range. This occurs even in networks where the traffic is not particularly high. Moreover, many of these collisions happen between control and data packets $(65 \%$ are control/data, $10 \%$ are data/data and $15 \%$ are among control packets).

Figure 2 shows the performance of DACAP when data/control collisions are possible and when they are not. The ideal case, termed DACAP-NC (no collisions), is a hypothetical case in which each node has the capability to suppress any control packets that arrive during reception of a data packet. The figure depicts the protocol throughput efficiency, i.e., the fraction of data bits correctly delivered to the destination, as a function of the data packet size for a varying offered load and two values of the channel bit error rate (BER). We observe that DACAP-NC always outperforms DACAP by a margin that can be quite significant in some cases. In particular, at the offered load of 0.3 packets per packet time, DACAP-NC correctly delivers up to $90 \%$ (80\%) of the data packets in scenarios with BER of $10^{-6}\left(10^{-4}\right)$, as compared to $60 \%$ (50\%) for DACAP.

To counteract the degradation caused by control/data packet collisions, we investigate the possibility to multiplex these two channels. Namely, we split the total available bandwidth into two bands, one for data and another for control packets. By doing so, the control/data collisions will be eliminated, leading to a reduction in the number of re-transmissions, but the bandwidth available for information transmission, i.e. the data rate, will be reduced. Hence, there is a trade-off involved in channel multiplexing, which we aim to assess through numerical analysis.

Earlier work on this topic includes [15], where the authors propose a protocol called Reservation Channel Acoustic Medium Access Protocol (RCAMAC). This solution, however, is analyzed only for the single-hop scenario. In [16], multiplexing is coupled with Aloha and a protocol based on RTS/CTS exchange. As in the RCAMAC case, the definition of these protocols is limited to the single-hop scenario.

In this paper, we focus on multi-hop scenarios, where random access achieves its full potential. We investigate the performance of DACAP with multiplexed control/data channels, which we call DACAP-M. Given a fixed available bandwidth, we first determine a suitable partition into data and control bands, including a guard band that is needed in practice to separate the two. Our simulation results show that despite the reduction in data bandwidth, such a solution yields performance improvement in terms of throughput efficiency and energy consumption.

The remainder of the paper is organized as follows. Section II describes channel multiplexing, i.e. bandwidth partitioning based on numerical optimization. Perfor- mance of DACAP-M is discussed in Section III and compared to that of DACAP. Concluding remarks are given in Section IV.

\section{BANDWIDTH PARTITIONING FOR CONTROL AND DATA CHANNELS}

Assuming that a fixed bandwidth is given, we want to partition it into the data and control bands, so as to satisfy some optimality criterion. The criterion that we consider is minimization of the total time it takes to one RTS/CTS/data/ACK exchange. By doing so, we aim to minimize the chances of collision, as shorter communications are less likely to collide. In turn, we expect the throughput to improve. In particular, we pose the optimization problem as follows:

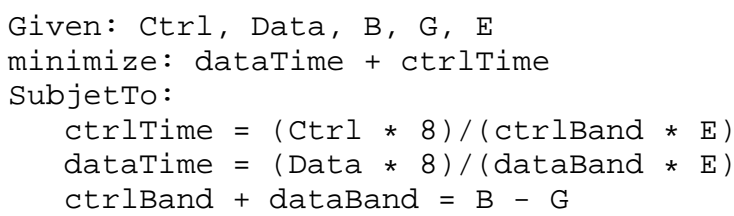

Here, Ctrl represents the total number of bytes in the RTS, CTS and ACK packets (including physical layer headers), Data represents the number of bytes in a data packet, $B$ is the available bandwidth, $G$ is the guard band, and $E$ is the bandwidth efficiency. We consider $\mathrm{B}=2000 \mathrm{~Hz}$, with a fixed guard band $\mathrm{G}=200 \mathrm{~Hz}$, and a bandwidth efficiency $E=1 \mathrm{bps} / \mathrm{Hz}$. The data packet payload varies from $50 \mathrm{~B}$ to $3000 \mathrm{~B}$, and the control payload sums up to $40 \mathrm{~B}$. Based on these settings, our program outputs a channel partitioning consisting of about $200 \mathrm{~Hz}$ for control packets and $1600 \mathrm{~Hz}$ for data packets in the majority of cases considered.

Using these values, in Section III we investigate the performance of DACAP-M. ${ }^{1}$ The particular questions that we want to answer are how well does this (realistic) implementation compare with (the ideal) DACAP-NC, and what performance gain, if any, does it offer with respect to conventional DACAP.

\section{PERFormance Evaluation}

The protocols were implemented in the VINT project ns-2 simulator [17] extended to include key characteristics of the underwater environment such as 3D deployment, propagation at the speed of sound, and acoustic path loss that depends on the distance and frequency.

\section{A. Simulation scenario}

We consider a scenario with 100 static nodes are placed on the seafloor at a depth of $200 \mathrm{~m}$. The nodes are randomly and uniformly scattered within a $4 \mathrm{~km} \times 4 \mathrm{~km}$ footprint. Packets are transmitted from the nodes to a

\footnotetext{
${ }^{1}$ Different ways of data/control bandwidth partitioning have also been tried, always yielding inferior performance.
} 


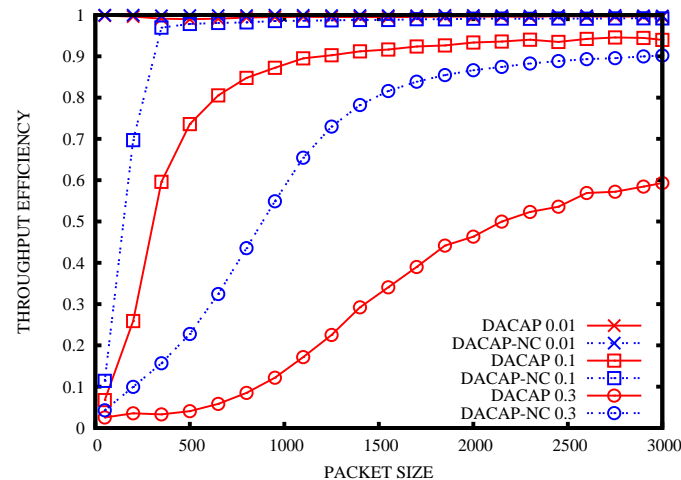

(a) $\mathrm{BER}=10^{-6}$

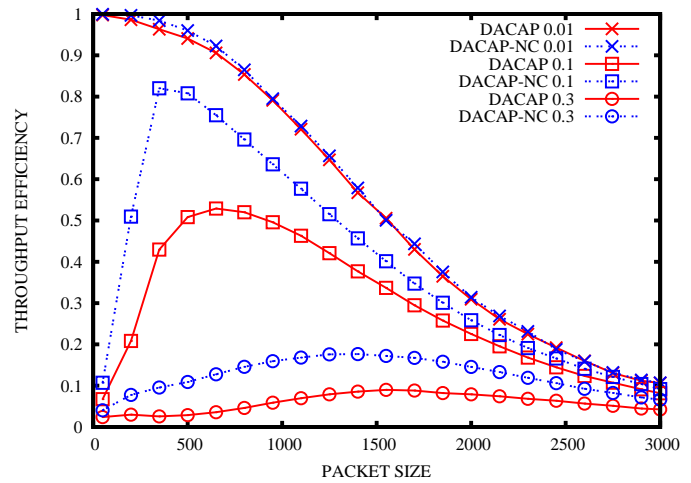

(b) $\mathrm{BER}=10^{-4}$

Figure 2. Comparing throughput efficiency in scenarios with and without control/data interferences. Legend indicates total offered load, measured in packets per packet time.

common sink (data collection point) located centrally on the surface.

All nodes have the same transmission radius $R$, whose value is set to $500 \mathrm{~m}, 1000 \mathrm{~m}$, or $1500 \mathrm{~m}$. Communication occurs in a multi-hop fashion, over shortest path routes, which are pre-determined for each $R$.

The receiving power and the idle power are set to $100 \mathrm{~mW}$. The transmission power, as well as the carrier frequency, are computed according to [18], so as to achieve the SNR of $20 \mathrm{~dB}$ for each $R$. The transmission power is listed in Tables I (DACAP) and II (DACAP-M).

Table I

TRANSMISSION POWER WITHOUT CHANNEL MULTIPLEXING

\begin{tabular}{|l|c|c|c|}
\hline$R$ & $500 \mathrm{~m}$ & $1000 \mathrm{~m}$ & $1500 \mathrm{~m}$ \\
\hline All packets & $1000 \mathrm{~mW}$ & $4930 \mathrm{~mW}$ & $13300 \mathrm{~mW}$ \\
\hline
\end{tabular}

Table II

TRANSMISSION POWER WITH CHANNEL MULTIPLEXING

\begin{tabular}{|l|c|c|c|}
\hline$R$ & $500 \mathrm{~m}$ & $1000 \mathrm{~m}$ & $1500 \mathrm{~m}$ \\
\hline Control packets & $200 \mathrm{~mW}$ & $600 \mathrm{~mW}$ & $1400 \mathrm{~mW}$ \\
Data packets & $860 \mathrm{~mW}$ & $4000 \mathrm{~mW}$ & $11000 \mathrm{~mW}$ \\
\hline
\end{tabular}

The data rate is 2000 bps without multiplexing, and 1600 bps with multiplexing. The data packets are thus $25 \%$ longer with multiplexing, and it takes 10 times longer to transmit control packets. Longer packets used with DACAP-M translate into higher transmission energy if the signal power is kept constant. In Table III we quantify the percentage of additional energy needed by DACAP-M to transmit one bit of data or control information.

The data payload size is chosen according to the channel BER and the offered load, so as to optimize the throughput efficiency [19]. Two different values of BER are considered, $10^{-6}$ and $10^{-4}$. For the lower BER,
Table III

DACAP-M: ADDITIONAL ENERGY PER BIT

\begin{tabular}{|l|c|c|c|}
\hline$R$ & $500 \mathrm{~m}$ & $1000 \mathrm{~m}$ & $1500 \mathrm{~m}$ \\
\hline Control & $100 \%$ & $22 \%$ & $5 \%$ \\
Data & $6 \%$ & $2 \%$ & $3 \%$ \\
\hline
\end{tabular}

the data payload is chosen as maximum, 3000 B. For the higher BER, where re-transmissions due to channel noise are more likely, shorter packets are preferred. The offered load now becomes important in the selection of packet sizes, which are listed in Table IV.

Table IV

PACKET SIZE FOR $B E R=10^{-4}$

\begin{tabular}{|l|c|c|c|}
\hline $\bar{\lambda} \downarrow \mid R \rightarrow$ & $500 \mathrm{~m}$ & $1000 \mathrm{~m}$ & $1500 \mathrm{~m}$ \\
\hline 0.01 & $50 \mathrm{~B}$ & $50 \mathrm{~B}$ & $50 \mathrm{~B}$ \\
0.04 & $200 \mathrm{~B}$ & $200 \mathrm{~B}$ & $200 \mathrm{~B}$ \\
0.07 & $350 \mathrm{~B}$ & $350 \mathrm{~B}$ & $500 \mathrm{~B}$ \\
0.1 & $500 \mathrm{~B}$ & $650 \mathrm{~B}$ & $800 \mathrm{~B}$ \\
0.3 & $1100 \mathrm{~B}$ & $1550 \mathrm{~B}$ & $1850 \mathrm{~B}$ \\
0.6 & $1400 \mathrm{~B}$ & $1100 \mathrm{~B}$ & $1250 \mathrm{~B}$ \\
\hline
\end{tabular}

Traffic is generated according to a Poisson process with aggregate (network-wide) rate $\lambda$ packets per seconds. We also define the normalized packet rate as $\bar{\lambda}=\lambda T_{\text {pack }}$, whose values are considered in the range 0 to 1 packets per packet time. The packet time used here is the one corresponding to transmission at full rate, i.e. $T_{\text {pack }}=N_{b} / R_{b}$, where where $N_{b}$ is the packet size in bits and $R_{b}=2000$ bps. Simulations were conducted for low traffic $(\bar{\lambda}<0.1)$, medium traffic $(\bar{\lambda}=0.3)$, and high traffic $(\bar{\lambda}=0.6)$.

Once a packet is generated it is associated with a source selected randomly among all the nodes. The destination of all the packets is the sink. The total size of the data packet is given by the payload plus the headers added by different layers (physical through 
network). The physical layer header contains all the information needed by the modem to start receiving a packet (synchronization preamble, delimiters, etc.). A synchronization peering time is taken to be $10 \mathrm{~ms}$. The MAC header contains the sender's ID, the destination's IDs and the packet type if needed. For both data and ACK packets, the MAC header length is set to $5 \mathrm{~B}$. The size of RTS and CTS packets is set to $8 \mathrm{~B}$.

To correctly receive each packet (control or data) the signal to interference ratio at the receiver is required to be SIR $\geq 15 \mathrm{~dB}$. Each node has a buffer of $30 \mathrm{~KB}$ where data coming from the upper layers are stored before transmission. Whenever the buffer is full and a new packet arrives, the oldest packet is discarded. We also limit the number of packets that can be stored to 50 , so that the nodes are not filling their buffers with old information. Our implementation of DACAP mandates to abandon RTS transmission after 7 failed attempts to access the channel, and to discard a data packet after 7 failed re-transmissions. Every point depicted in our figures has been obtained by averaging over the number of simulations needed to achieve a statistical confidence of $95 \%$ with a $5 \%$ precision.

\section{B. Performance metrics}

The following metrics have been used to assess the system performance.

1) Throughput efficiency, defined as the ratio between the bit rate delivered to the sink (correct bits) and the bit rate offered to the network, $N_{b} \lambda$.

2) Energy per bit, defined as the energy consumed by the network to correctly deliver a bit of data to the sink.

3) End-to-end latency per meter, defined as the time between when a packet is generated and the time when it is correctly received by the sink, divided by the distance between the source and destination. Normalization by the distance is used to unify the performance over a varying coverage area (a large area will entail proportionately large propagation delay). This metric is computed only for the packets correctly delivered, and averaged over all the packets.

4) Route length, defined as the average number of hops traversed by packets correctly delivered to the sink.

In what follows we discuss these performance measures as function of the offered load, considering different transmission ranges $R$, and different BERs.

\section{Performance Analysis}

1) Throughput efficiency is shown in Figures 3(a) and 3(b). These figures indicate that advantages are indeed to be had from control/data multiplexing. In all situations considered, DACAP-M outperforms DACAP (except, perhaps, at low BER, low traffic, and short transmission range, where they are comparable). For each $(R, \mathrm{BER})$ combination, there is a window of offered loads for which the improvement is most notable. This window falls between the very low traffic (where both protocols perform equally well) and some value of the offered load after which both protocols reach saturation as they fail to cope with the increasing traffic. The exact amount of improvement depends on the particular $(R$, BER) pair, and also on the offered load. For example, at $\left(1000 \mathrm{~m}, 10^{-6}\right)$ and $\bar{\lambda}=0.3$, DACAP-M yields $27 \%$ more in throughput efficiency compared to DACAP which achieves an efficiency of 0.6.

2) Energy consumption per bit is shown in Figures 4(a) and 4(b). These results reveal another advantage of multiplexing, namely the fact that elimination of data/control collisions reduces the overall energy consumption, despite the fact that longer transmission times require more energy (Table III). Similarly as with the throughput efficiency, DACAP-M outperforms DACAP in all the situations considered. The greatest advantage, however, comes in situations with high traffic. In these situations, the throughput efficiency of both protocols suffers as they become saturated, but whereas DACAP's energy consumption rapidly increases as the protocol is trying to push the data packets through the system, DACAP-M saves the energy that would be wasted in retransmitting all those data packets that were intercepted by control packets.

For example, at a BER $=10^{-6}$, energy per bit consumption at high traffic improves by $22 \%, 36 \%$, and $170 \%$ for the transmission range of $500 \mathrm{~m}, 1000 \mathrm{~m}$ and $1500 \mathrm{~m}$, respectively.

3) Packet latency per meter is shown in Figure 5(a) and 5(b). There are multiple trends involved in the delay performance, and the two protocols show different delay behavior depending upon the BER and the offered load. In general, so long as the system is below the saturation point, DACAP-M exhibits longer delays. This penalty seems to be greater for shorter transmission ranges, which results in longer routes. As the traffic increases, the delay of both protocols increases, but the rate of increase depends on the transmission radius, and is lower for DACAP-M, thus giving it advantage at greater $R$. This effect is especially noticeable at low BER, and can be explained by the fact that fewer hops incur less processing delay. Hence, although DACAP-M uses longer packets that take longer to process, the overall delay is less if fewer hops are used. At high BER, many packet losses are due to the channel noise and not only collisions, and, therefore, multiplexing looses part of its effectiveness.

4) Route length is shown in Figure 6. The average number of hops is seen to decrease with traffic, following a similar trend for both protocols. It is interesting to note that a node is on the average 7 hops away from 


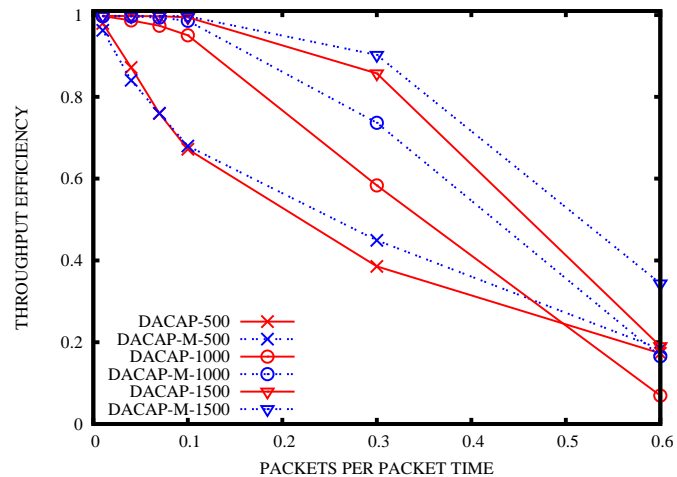

(a) $\mathrm{BER}=10^{-6}$

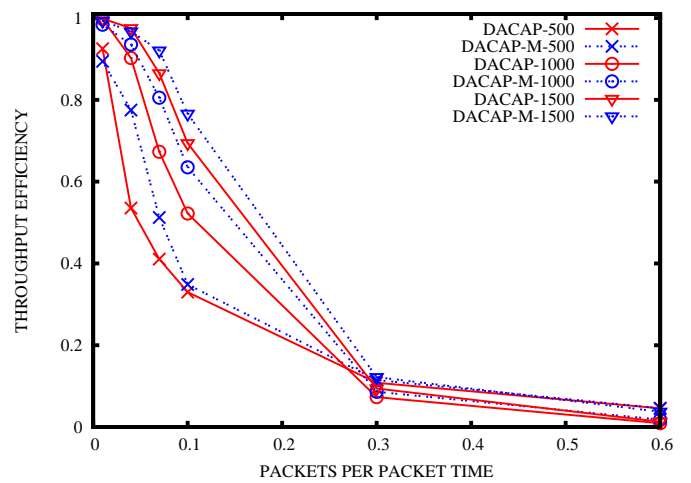

(b) $\mathrm{BER}=10^{-4}$

Figure 3. Throughput efficiency.

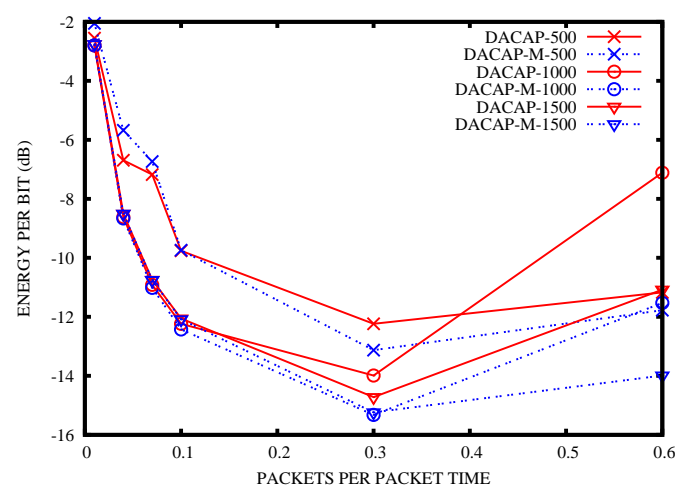

(a) $\mathrm{BER}=10^{-6}$

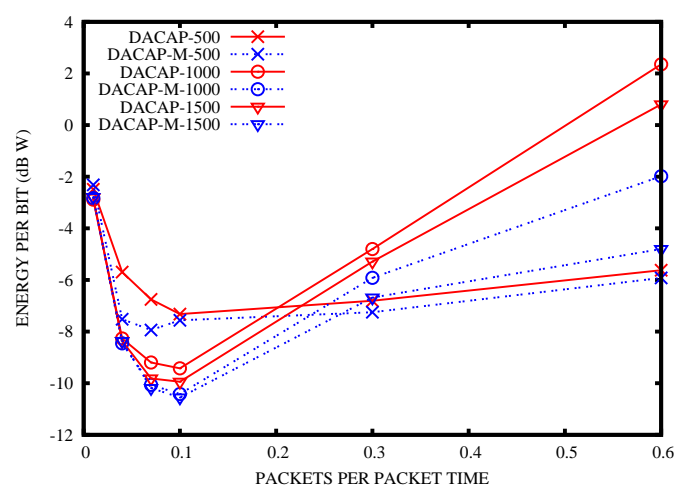

(b) $\mathrm{BER}=10^{-4}$

Figure 4. Energy consumption per bit.

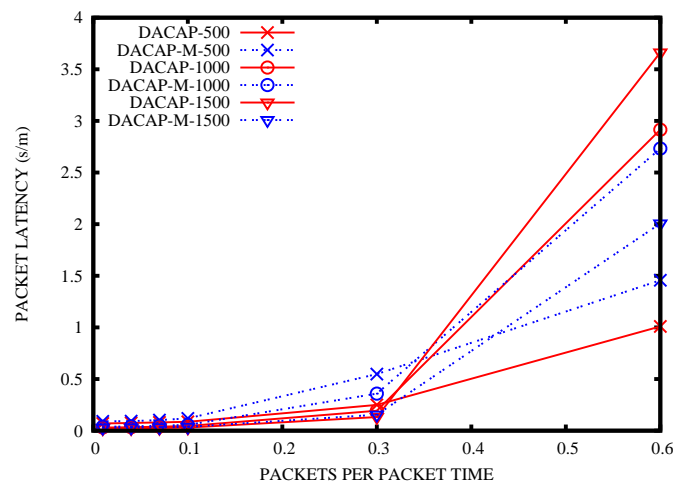

(a) $\mathrm{BER}=10^{-6}$

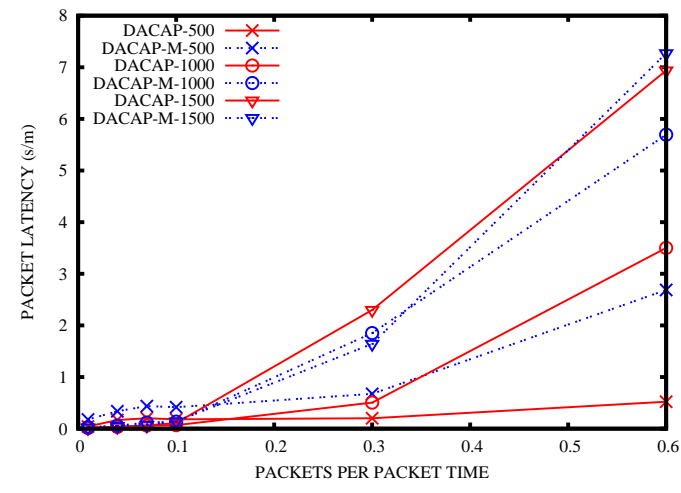

(b) $\mathrm{BER}=10^{-4}$

Figure 5. Packet latency per meter.

the sink at $R=500 \mathrm{~m}, 2.3$ at $1000 \mathrm{~m}$, and 1.5 at $1500 \mathrm{~m}$, but that the average number of hops traveled by a successful packet, when the traffic is high, is less than this quantity. This means that as the number of re-transmissions increases and the network becomes congested, nodes closer to the sink are those that deliver more packets successfully. Packets from nodes farther away are more likely to be lost. This effect is most pronounced for short transmission range, and can be used to support some of the observations made regarding the latency.

5) Comparison with ideal case (DACAP-NC). We conclude this section by comparing the performance of DACAP-M with the ideal case, i.e., the performance of 


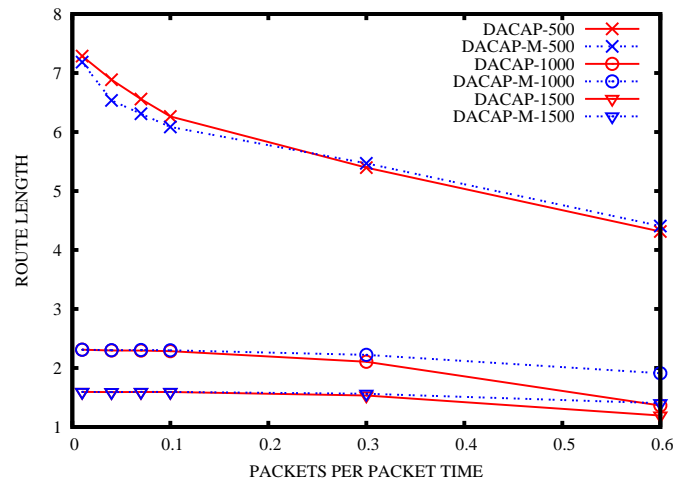

(a) $\mathrm{BER}=10^{-6}$

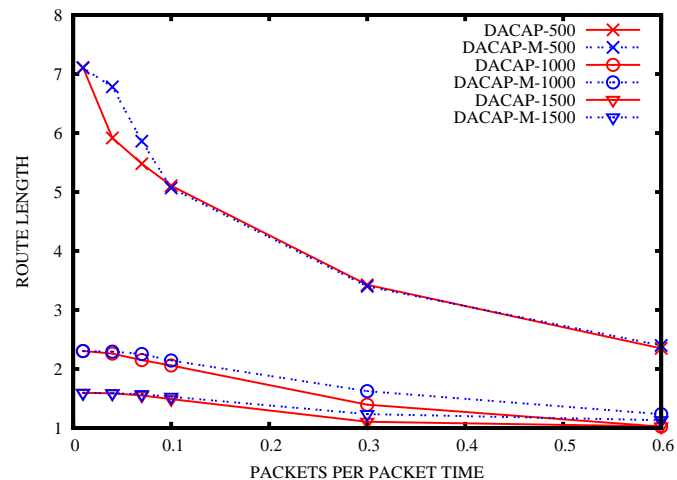

(b) $\mathrm{BER}=10^{-4}$

Figure 6. Length of the routes traveled by packets correctly delivered to the sink.

DACAP with no collisions between data and control packet (DACAP-NC, as discussed in Section I). Figure 7 shows the throughput efficiency comparison for the case of $1000 \mathrm{~m}$ transmission radius. The performance of DACAP-M is below that of DACAP-NC because of the loss in data $\mathrm{b}$ bandwidth (bit rate) that results from multiplexing. For the present system parameters, the loss in throughput efficiency is below $22 \%$ for DACAPM (54\% for DACAP). At low traffic rates, however, DACAP-M approaches the performance of DACAP-NC.

\section{Conclusions}

Multiplexing of the data and control channels was considered for random access underwater networks that use MAC protocols based on RTS/CTS exchange. The goal in doing so was to eliminate the collisions between the data and control packets, which have been shown to cause a non-negligible loss in throughput. Allocating a portion of the bandwidth exclusively to the control channel results in improved packet delivery at the price of a lower data rate. To assess the trade-offs involved, a simulation analysis was conducted. We focused on a particular MAC protocol, the delay-aware collision avoidance protocol (DACAP), which we coupled with channel multiplexing (DACAP-M).

Numerical results show that multiplexing offers some improvement in both the throughput efficiency and the energy per bit consumption, i.e., that the benefits of eliminating control/data collisions outweigh the reduction in the data bandwidth. ¿From the viewpoint of delay, there is a trade-off in choosing whether to multiplex or not, and prolonged packet durations in general imply some penalty for multiplexing. However, the fact that this penalty is small gives precedence to the throughput and energy benefits, which are the more critical figures of merit in the vast majority of underwater acoustic systems.

\section{ACKNOWLEDGMENTS}

This work was partially supported by the International FIRB contract RBIN047MH9 and the NSF grant 0708420.

\section{REFERENCES}

[1] I. F. Akyildiz, D. Pompili, and T. Melodia, "Underwater acoustic sensor networks: Research challenges," Elsevier's Journal of Ad Hoc Networks, vol. 3, no. 3, pp. 257-279, May 2005.

[2] J.-H. Cui, J. Kong, G. M., and Z. S., "Challenges: Building scalable mobile underwater wireless sensor networks for aquatic applications," IEEE Network, Special Issue on Wireless Sensor Networking, vol. 20, no. 3, pp. 12-18, May 2006.

[3] J. Heidemann, W. Ye, J. Willis, A. A. Syed, and Y. Li, "Research challenges and applications for underwater sensor networking," in Proceedings of the IEEE Wireless Communications and Networking Conference, WCNC 2006, Las Vegas, NV, April 3-6 2006, pp. 229-235.

[4] J. Partan, J. Kurose, and B. N. Levine, "A survey of practical issues in underwater networks," in WUWNet '06: Proceedings of the 1st ACM international workshop on Underwater networks. New York, NY, USA: ACM, September 2006, pp. 17-24.

[5] L. Lanbo, Z. Shengli, and C. Jun-Hong, "Prospects and problems of wireless communication for underwater sensor networks," Wireless Communications and Mobile Computing, Special Issue on Underwater Sensor Networks, vol. 8, no. 8, pp. 977-994, August 2008.

[6] I. F. Akyildiz, D. Pompili, and T. Melodia, "State of the art in protocol research for underwater acoustic sensor networks," SIGMOBILE Mobile Computing Communication Review, vol. 11, no. 4, pp. 11-22, October 2007.

[7] J. Yackoski and C. Shen, "UW-FLASHR: Achieving high channel utilization in a time-based acoustic MAC protocol," in $\mathrm{WuWNeT}$ '08: Proceedings of the third ACM international workshop on Wireless network testbeds, experimental evaluation and characterization, San Francisco, California, USA, September 15 2008, pp. 59-66.

[8] N. Chirdchoo, W.-S. Soh, and K. C. Chua, "ALOHA-based MAC protocols with collision avoidance for underwater acoustic networks," in Proceedings of IEEE INFOCOM 2007, the 26th IEEE International Conference on Computer Communications, Anchorage , Alaska , USA, May 6-12 2007.

[9] M. Molins and M. Stojanovic, "Slotted FAMA: A MAC protocol for underwater acoustic networks," in Proceedings of MTS/IEEE OCEANS 2006, 2006. 


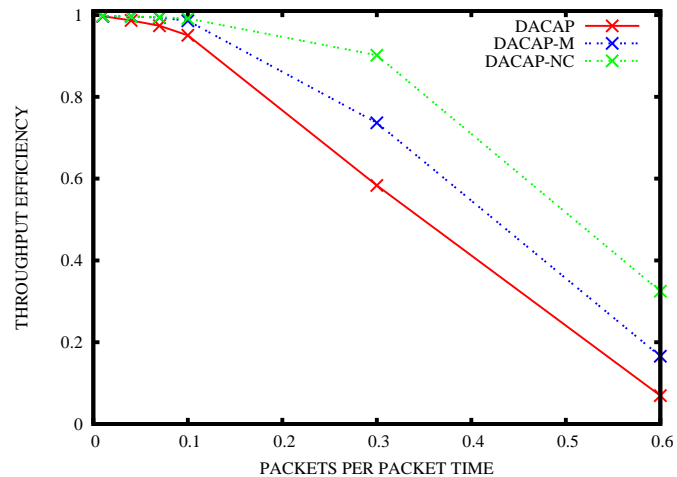

(a) $\mathrm{BER}=10^{-6}$

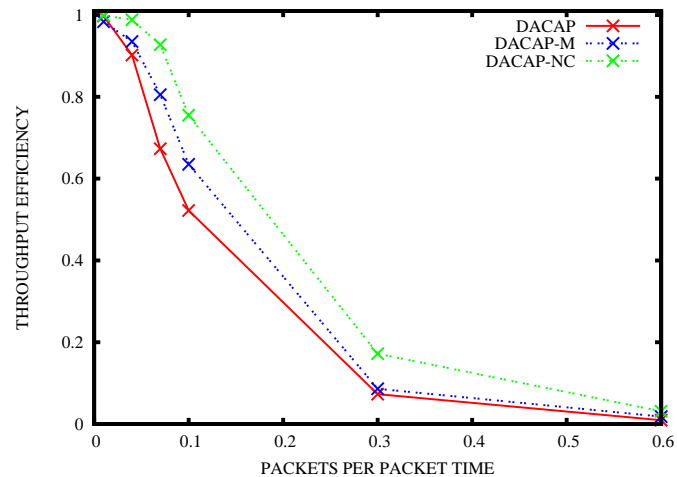

(b) $\mathrm{BER}=10^{-4}$

Figure 7. DACAP-NC, DACAP and DACAP-M vs. throughput efficiency: A Comparison $(R=1000)$.

[10] B. Peleato and M. Stojanovic, "Distance aware collision avoidance protocol for ad-hoc underwater acoustic sensor networks." IEEE Communications Letters, vol. 11, no. 12, pp. 1025-1027, December 2007.

[11] V. Rodoplu and M. Park, "An energy-efficient MAC protocol for underwater wireless acoustic networks," in Proceedings of MTS/IEEE OCEANS 2005, 2005.

[12] X. Guo, M. Frater, and M. Ryan, "An adaptive propagation-delaytolerant MAC protocol for underwater acoustic sensor networks," OCEANS 2007 - Europe, June 18-21 2007.

[13] A. Syed, W. Ye, and J. Heidemann, "Comparison and evaluation of the T-Lohi MAC for underwater acoustic sensor networks," IEEE Journal on Selected Areas in Communications, vol. 26, no. 9, pp. 1731-1743, December 2008.

[14] C. Petrioli, R. Petroccia, and M. Stoanovic, "A comparative performance evaluation of MAC protocols for underwater sensor networks." in Proceedings of MTS/IEEE OCEANS 2008, Quebec City, Quebec, Canada, September 15-18 2008.

[15] L. T. Tracy and S. Roy, "A reservation MAC protocol for ad- hoc underwater acoustic sensor networks," in WuWNeT '08: Proceedings of the third ACM international workshop on Wireless network testbeds, experimental evaluation and characterization, San Francisco, California, USA, September 15 2008, pp. 95-98.

[16] Z. Zhou, P. Zheng, J. H. Cui, and S. Zhjie, "Analyzing multichannel MAC protocols for underwater acoustic sensor networks," UCONN CSE Technical Report: UbiNet-TR08-02, January 2009.

[17] The VINT Project, The ns Manual. http://www.isi.edu/nsnam/ns/, 2002.

[18] M. Stojanovic, "On the relationship between capacity and distance in an underwater acoustic channel," in Proceedings of the First ACM International Workshop on UnderWater Networks, WuWNet 2006, Los Angeles, CA, September 252006.

[19] S. Basagni, C. Petrioli, R. Petroccia, and M. Stoanovic, "Choosing the packet size in multi-hop underwater networks," Technical Report 06/2009, Dipartimento di Informatica, Università di Roma "La Sapienza", Rome, Italy, June 2009. 